\title{
A functional of the one-body-reduced density matrix derived from the homogeneous electron gas: Performance for finite systems
}

\author{
N. N. Lathiotakis, ${ }^{1,2,3}$ N. Helbig, ${ }^{2,3,4,5}$ A. Zacarias, ${ }^{2,3}$ and E. K. U. Gross ${ }^{2,3}$ \\ ${ }^{1}$ Theoretical and Physical Chemistry Institute, NHRF, \\ Vas. Konstantinou 48, GR-11635, Athens, Greece \\ ${ }^{2}$ Institut für Theoretische Physik, Freie Universität Berlin, Arnimallee 14, D-14195 Berlin, Germany \\ ${ }^{3}$ European Theoretical Spectroscopy Facility \\ ${ }^{4}$ Fritz-Haber-Institut der Max-Planck-Gesellschaft, Faradayweg 4-6, 14195 Berlin, Germany \\ ${ }^{5}$ Unité de Physico-Chimie et de Physique des Materiéaux, \\ Université Catholique de Louvain, B-1348 Louvain-la-Neuve, Belgium
}

\begin{abstract}
An approximation for the exchange-correlation energy of reduced-density-matrix-functional theory was recently derived from a study of the homogeneous electron gas (N.N. Lathiotakis, N. Helbig, E.K.U. Gross, Phys. Rev. B 75, 195120 (2007)). In the present work, we show how this approximation can be extended appropriately to finite systems, where the Wigner Seitz radius $r_{s}$, the parameter characterizing the constant density of the electron gas, needs to be replaced. We apply the functional to a variety of molecules at their equilibrium geometry, and also discuss its performance at the dissociation limit. We demonstrate that, although originally derived from the uniform gas, the approximation performs remarkably well for finite systems.
\end{abstract}

PACS numbers:

\section{INTRODUCTION}

Density-functional theory (DFT) is one of the most widely applied tools for electronic structure calculations. While it successfully describes systems ranging from atoms and molecules to solids, present day DFT approximations fail to describe a class of systems generally called strongly correlated. For these systems, recent calculations have fueled the hope that reduced-density-matrixfunctional theory (RDMFT) can cure the problem. ${ }^{1-3}$ Within RDMFT, the one-body reduced density matrix (1-RDM), $\gamma\left(\mathbf{r}, \mathbf{r}^{\prime}\right)$, is used as the basic variable in analogy to DFT, where that role is reserved for the electronic density. The theorem of Gilbert ${ }^{4}$, which is an extension of the Hohenberg-Kohn theorem to non-local external potentials, guarantees that the ground-state expectation value of any observable of a quantum mechanical system is a unique functional of the ground-state 1-RDM. In particular, the total energy $E_{\text {tot }}$ of a system of $N$ electrons moving in an external local potential $V(\mathbf{r})$ can be written in terms of the ground-state $\gamma$, as

$$
\begin{aligned}
E_{\mathrm{tot}}[\gamma]= & \iint d^{3} r d^{3} r^{\prime} \delta\left(\mathbf{r}-\mathbf{r}^{\prime}\right)\left[-\frac{1}{2} \nabla_{\mathbf{r}}^{2}+V(\mathbf{r})\right] \gamma\left(\mathbf{r}, \mathbf{r}^{\prime}\right) \\
& +\frac{1}{2} \iint d^{3} r d^{3} r^{\prime} \frac{\gamma(\mathbf{r}, \mathbf{r}) \gamma\left(\mathbf{r}^{\prime}, \mathbf{r}^{\prime}\right)}{\left|\mathbf{r}-\mathbf{r}^{\prime}\right|}+E_{\mathrm{xc}}[\gamma]
\end{aligned}
$$

(atomic units are used throughout). The first term contains the kinetic and external energies and is a simple functional of $\gamma$. The fact that the kinetic energy can be written explicitly in terms of the ground-state 1RDM is a great advantage of RDMFT compared to DFT. The energy contribution associated with the electronelectron interaction can be cast into two terms, the direct Coulomb energy (or Hartree) term which is again an explicit functional of $\gamma$, and the remaining contribution which is called exchange-correlation (xc) energy. The $\mathrm{xc}$ energy is an unknown functional of the 1-RDM and needs to be approximated in practice. Contrary to DFT, however, it does not contain any kinetic energy contributions but is solely given as the difference between the full Coulomb interaction and the Hartree energy. Several approximations for the xc energy have been introduced so far ${ }^{1-3,5-16}$, the great majority of them being implicit functionals of the 1-RDM. They depend explicitly on the natural orbitals $\varphi_{j}$ and the corresponding occupation numbers $n_{j}$, i.e. the eigenfunctions and the eigenvalues of $\gamma$, which are given by

$$
\int d^{3} r^{\prime} \gamma\left(\mathbf{r}, \mathbf{r}^{\prime}\right) \varphi_{j}\left(\mathbf{r}^{\prime}\right)=n_{j} \varphi_{j}(\mathbf{r})
$$

Applications of different RDMFT functionals for the calculation of the dissociation of molecules ${ }^{2,17}$ the ionization potential $^{18-21}$, or the fundamental gap ${ }^{21,22}$, have been reported. Most approximate RDMFT functionals can be written in the form

$$
\begin{aligned}
& \quad E_{\mathrm{xc}}[\gamma]=E_{\mathrm{xc}}\left[\left\{n_{j}\right\},\left\{\varphi_{j}\right\}\right]= \\
& -\frac{1}{2} \sum_{j, l=1}^{\infty} f\left(n_{j}, n_{l}\right) \iint d^{3} r d^{3} r^{\prime} \frac{\varphi_{j}^{*}(\mathbf{r}) \varphi_{l}^{*}\left(\mathbf{r}^{\prime}\right) \varphi_{l}(\mathbf{r}) \varphi_{j}\left(\mathbf{r}^{\prime}\right)}{\left|\mathbf{r}-\mathbf{r}^{\prime}\right|}
\end{aligned}
$$

with some function $f\left(n_{j}, n_{l}\right)$ which distinguishes these approximations from the Hartree-Fock approximation.

The first approximation of the kind in Eq. (3) was introduced by Müller ${ }^{5}$ using the function $f\left(n_{j}, n_{l}\right)=$ $\sqrt{n_{j} n_{l}}$. Buijse and Baerends rederived the same approximation from modeling exchange and correlation holes. ${ }^{1}$ The Müller functional overcorrelates in all systems it was applied to. ${ }^{23-28}$ Interestingly, for diatomic molecules, it reproduces the dissociation into two independent atomic 
fragments correctly. Goedecker and $\operatorname{Umrigar}^{6}$ (GU) considered a functional with the same function $f$ but excluding explicitly the self-interaction terms, $j=l$, in Eq. (3) and in the direct Coulomb term in Eq. (1). They used their functional in a minimization procedure and determined the natural orbitals and the corresponding occupation numbers. In this way, they found correlation energies, for small atomic and molecular systems, that are in very good agreement with the exact ones. However, it was found later that the GU functional fails to describe the dissociation of diatomic molecules correctly ${ }^{23,24}$. In an attempt to correct the overcorrelation of the Müller functional, while keeping the good description in the dissociation limit, Gritsenko et al. ${ }^{2}$ proposed a hierarchy of three levels of repulsive corrections. All three corrections distinguish between weakly and strongly occupied orbitals, the former being orbitals with occupation numbers close to zero, the latter having occupations close to one. The resulting functionals are called $\mathrm{BBC} 1, \mathrm{BBC} 2$, and $\mathrm{BBC} 3$. For the first two approximations the function $f$ is given by

$$
\begin{gathered}
f^{\mathrm{BBC} 1}\left(n_{j}, n_{l}\right)=\left\{\begin{array}{cl}
-\sqrt{n_{j} n_{l}}, & j \neq l, \text { and } \\
\sqrt{n_{j} n_{l}}, & j, l \text { weakly occupied },
\end{array}\right. \\
f^{\mathrm{BBC} 2}\left(n_{j}, n_{l}\right)=\left\{\begin{array}{cl}
-\sqrt{n_{j} n_{l}}, & j \neq l, \text { and } \\
& j, l \text { weakly occupied }, \\
n_{j} n_{l}, & j \neq l, \text { and } \\
& j, l \text { strongly occupied }, \\
\sqrt{n_{j} n_{l},} & \text { otherwise. }
\end{array}\right.
\end{gathered}
$$

In the BBC3 functional, the anti-bonding orbital is treated as strongly occupied. Additionally, the selfinteraction terms are removed as in the GU functional, except for the pair of bonding and anti-bonding orbitals. Gritsenko et al used the BBC functionals to calculate the dissociation curves of diatomic molecules. They concluded that the BBC3 functional is very accurate in the description of these systems both at the equilibrium geometry and the dissociation limit. Two other functionals, derived from a cumulant expansion of the second order density matrix, with a final form that is very similar to the BBC functionals were introduced by Piris. ${ }^{14} \mathrm{We}$ refer to these functionals as Piris natural orbital functionals. The first approximation, PNOF0, is identical to the $\mathrm{BBC} 1$ functional apart from the self-interaction terms which are removed in the same way as in the GU approximation. In the second functional, PNOF, an additional term is included to avoid occupation numbers which are identical to zero or one. PNOF0 and PNOF coincide for two electron systems. The BBC as well as PNOF and PNOF0 functionals were evaluated recently for a large set of molecular systems and were proven to be quite accurate in reproducing the correlation and the atomization energies of these systems. ${ }^{25}$

For the application of 1-RDM functionals to periodic systems, the homogeneous electron gas (HEG) is an im- portant prototype system. Also, as far as size is concerned, the HEG and small atomic and molecular systems are two opposite extremes. For the HEG, the GU and Müller functionals are identical since the self-interaction terms vanish. Similarly, all terms that include a special treatment of single orbitals vanish. As a result, the $\mathrm{BBC} 3$ functional coincides with $\mathrm{BBC} 2$, and $\mathrm{PNOF} 0$ with $\mathrm{BBC} 1$ in this special case. Cioslowski and Pernal ${ }^{26}$ as well as Csányi and Arias ${ }^{27}$ applied the Müller functional to the HEG. As for finite systems, the correlation energy is overestimated, actually by a factor of about two in the high density regime. In the low density region, the Müller functional fails completely to reproduce the limit of zero correlation. ${ }^{26-28}$ Csányi and Arias also introduced a different functional starting from a tensor expansion of the second-order matrix. Unfortunately, it fails to describe the electronic correlation of the HEG in both the dense and dilute limits. A more successful functional for the HEG is the one proposed by Csányi, Goedecker and Arias $(\mathrm{CGA})^{7}$, which reproduces relatively accurately the correlation energy for the dense HEG. Recently, we applied the $\mathrm{BBC} 1$ and $\mathrm{BBC} 2$ functionals to the $\mathrm{HEG}^{28}$. We showed that these functionals offer a better description of the correlation of the HEG over the whole range of densities than any other of the discussed functionals. Both the $\mathrm{BBC} 1$ and the $\mathrm{BBC} 2$ functionals overcorrelate slightly for high densities and undercorrelate for low densities. The crossover is at around $r_{s}=0.5$ where these functionals perform best. Additionally, they produce a finite discontinuity in the momentum distribution at the Fermi energy, resembling a feature of the exact theory. Unfortunately, the size and the dependence on the density of this discontinuity are not in agreement with the exact result.

\section{THE BBC ++ FUNCTIONAL}

In an attempt to improve over the $\mathrm{BBC}$ functionals for the HEG, we introduced a modification to the BBC1 functional ${ }^{28}$. It consists of the introduction of a parameter into the function $f$ fitted for each value of $r_{s}$ to reproduce the exact correlation energy of the HEG. Two choices for this parameter were made. We concluded that a reasonable way to generalize $\mathrm{BBC} 1$ is to introduce a function $s\left(r_{s}\right)$ multiplying the function $f$ when both orbitals are weakly occupied. The corresponding function $f$ then reads

$$
f\left(n_{j}, n_{l}\right)=\left\{\begin{array}{cl}
-s\left(r_{s}\right) \sqrt{n_{j} n_{l}}, & j \neq l, \text { and } \\
& j, l \text { weakly occupied }, \\
\sqrt{n_{j} n_{l}}, & \text { otherwise. }
\end{array}\right.
$$

We call this functional $s$-functional. Note that, for the HEG, if one assumes plane-wave natural orbitals, no special care is necessary for the $j=l$ terms since these terms vanish. The $s$-functional reproduces, by construction, the exact correlation energy of the HEG. It also improves the 


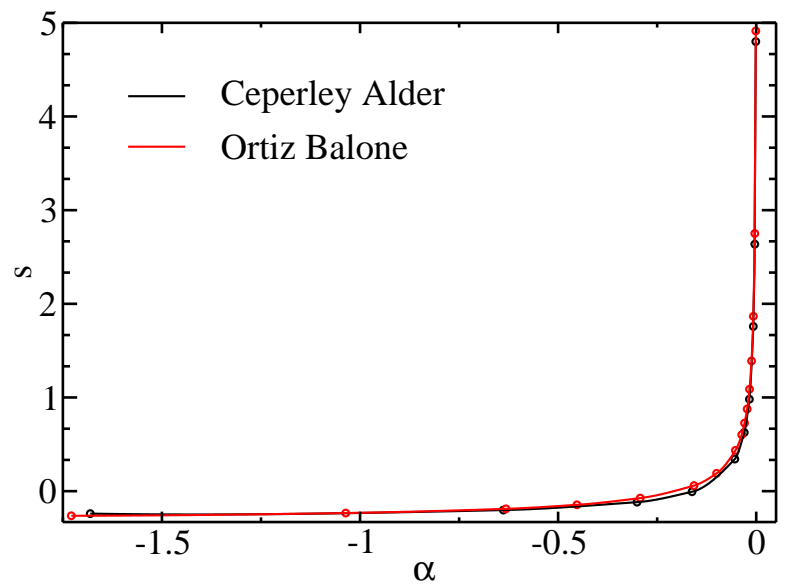

FIG. 1: Dependence of the fitting parameter $s$ of the $s$ functional $^{28}$ on the ratio $\alpha$ of the correlation over the kinetic energy for the HEG. Two different functions $s$ are plotted for two sets of diffusion Monte-Carlo data which $s$ was fitted to reproduce: From Ceperley and Alder ${ }^{29}$ and Ortiz and Balone $^{30}$.

calculated momentum distribution compared to BBC1 and $\mathrm{BBC}$. However, the momentum distribution still deviates from the exact one ${ }^{28}$.

Being derived from the study of the HEG, the $s$ functional is directly applicable to metallic systems, where a value of $r_{s}$ can be associated. The application to other systems is not straightforward since one has to overcome the $r_{s}$ dependence. One possibility, in the spirit of the local density approximation of DFT, is to express $s$ as a function of the local electronic density. Hence, $s$ becomes a function of the space coordinate $\mathbf{r}$ and therefore should be properly included in the integrals in Eq. (3). A reasonable choice that preserves the symmetry of the integrations over $\mathbf{r}$ and $\mathbf{r}^{\prime}$ is to multiply the integrand in Eq. (3) by $\sqrt{s(n(\mathbf{r})) s\left(n\left(\mathbf{r}^{\prime}\right)\right)}$ or other possible averages and keep the $\mathrm{BBC} 1$ form for the function $f$. Work investigating the performance of the resulting functional is in progress.

In the present article, we propose an alternative way to circumvent the $r_{s}$ dependence. We refer to the resulting functional as the $\mathrm{BBC}++$ functional. In this approximation, the xc terms retain the simple form of the exchange integrals over Gaussian or Slater type orbitals. The idea is to establish the dependence of $s$ on a quantity $\alpha$ which, contrary to $r_{s}$, is meaningful for all systems, finite and periodic, metallic and insulating. We choose the ratio of the correlation energy over the Hartree-Fock kinetic energy for this quantity $\alpha$. For the HEG, $\alpha$ depends on the density parameter $r_{s}$ and, since the function $\alpha\left(r_{s}\right)$ is strictly monotonic and therefore invertible, the dependence $s(\alpha)$ can be established. The resulting function $s(\alpha)$ for the HEG is shown in Fig. 1. The function $s\left(r_{s}\right)$, which is necessary in the calculation of $s(\alpha)$, is given in Ref. 28. The difficulty for the application of the $\mathrm{BBC}++$ functional lies in the fact that the correlation energy, and consequently $\alpha$, are only known at the solution point, i.e. when the optimal $\gamma$ is known. Thus, $s(\alpha)$ has to be determined self-consistently during the minimization procedure. Starting from a trial value for $s$ one minimizes the energy with respect to $\gamma$, calculates $\alpha$ and feeds the corresponding value for $s$ back into the functional. As the $\mathrm{BBC}++$ functional coincides with the $s$-functional for the HEG, the self-consistent determination of $s(\alpha)$ has to yield the correct $s\left(r_{s}\right)$ in this case. Using the implementation for the HEG presented in Ref. 28, we verified that this is indeed the case. We also found that the selfconsistent determination of $s$ converges for all the finite systems we studied.

\section{RESULTS FOR FINITE SYSTEMS}

For the application of 1-RDM functionals to finite systems, we used the implementation introduced in Ref. 12 which relies on the GAMESS computer program ${ }^{31}$ for the calculation of the one- and two- electron integrals. In the present work, we employed the cc-pVTZ basis set $^{32}$ for all systems apart from the He atom for which the ccpVQZ set ${ }^{33}$ was used. Depending on the system, these basis sets contain 30 to 50 basis functions. We always made full use of the size of the basis set, optimizing as many natural orbitals as there were basis functions available. For the BBC3 functional, we used the form that respects the possible degeneracies of the bonding and anti-bonding orbitals. ${ }^{25}$ The total energies resulting from this full minimization with respect to the natural orbitals and occupation numbers for several atoms and diatomic molecules are given in Table I. We compare all our results to total energies obtained from a coupled-cluster-singlesdoubles-triples $(\operatorname{CCSD}(\mathrm{T}))^{34}$ calculation using the Gaussian 03 computer program ${ }^{35}$ with the same basis sets as used in the RDMFT calculation. For the systems considered here, the $\mathrm{BBC}++$ functional yields slightly better total energies than GU but does not reach the accuracy of the BBC3 and Piris functionals. However, it performs significantly better than the $\mathrm{BBC} 1$ approximation that it was derived from, except for the $\mathrm{H}_{2}$ molecule. The repulsive correction to the $\mathrm{BBC} 1$ functional increases with increasing number of electrons in the system. Overall, for small finite systems, the $\mathrm{BBC}++$ functional performs remarkably well considering that it was originally tuned to be exact for the HEG.

In Fig. 2, we plot the dissociation curve for the $\mathrm{H}_{2}$ molecule. As already mentioned, among all the systems we studied, $\mathrm{H}_{2}$ is the only case where the $\mathrm{BBC}++$ functional does not improve over BBC1. Nevertheless, $\mathrm{BBC}++$ reproduces the dissociation curve surprisingly well giving a qualitatively correct optimal 1-RDM with four occupations equal to 0.5 (per spin) in the limit of large distance. The slight decrease in the total energy at around $2.5 \AA$ separation, which can be seen in Fig. 2, is a pathology of the $\mathrm{BBC}++$ functional. It originates from the dramatic increase in static correlation leading 


\begin{tabular}{c|cccccc|c}
\hline \hline Functional & $\mathrm{He}$ & $\mathrm{Be}$ & $\mathrm{H}_{2}$ & $\mathrm{LiH}$ & $\mathrm{Ne}$ & $\mathrm{HF}$ & $\bar{\Delta}$ \\
\hline Hartree-Fock & 2.8615 & 14.5729 & 1.1330 & 7.9868 & 128.5320 & 100.0589 & 0.119 \\
Müller & 2.9143 & 14.7471 & 1.1905 & 8.1167 & 128.9168 & 100.5185 & 0.090 \\
GU & 2.8980 & 14.6456 & 1.1660 & 8.0478 & 128.8260 & 100.3692 & 0.019 \\
BBC1 & 2.9042 & 14.6692 & 1.1746 & 8.0642 & 128.8471 & 100.4105 & 0.035 \\
BBC3 & 2.9005 & 14.6490 & 1.1705 & 8.0444 & 128.8014 & 100.3373 & 0.011 \\
PNOF & 2.8925 & 14.6234 & 1.1593 & 8.0310 & 128.7876 & 100.3166 & 0.013 \\
PNOF0 & 2.8925 & 14.6236 & 1.1593 & 8.0309 & 128.7840 & 100.3133 & 0.014 \\
BBC++ & 2.9035 & 14.6574 & 1.1768 & 8.0572 & 128.7862 & 100.3526 & 0.018 \\
CCSD $(\mathrm{T})$ & 2.9025 & 14.6186 & 1.1724 & 8.0227 & 128.8054 & 100.3401 & 0.000 \\
\hline$s$ & 1.202 & 2.108 & 0.573 & 1.674 & 3.158 & 2.823 & \\
$\alpha$ & -0.0147 & -0.00580 & -0.0388 & -0.00882 & -0.00198 & -0.00344 & \\
\hline \hline
\end{tabular}

TABLE I: Absolute total energies (atomic units) of atoms and diatomic molecules calculated with different 1-RDM functionals and the average absolute deviation $\bar{\Delta}$ from the reference energies obtained with the $\operatorname{CCSD}(\mathrm{T})^{34}$ method. For the latter, we used the Gaussian 03 program $^{35}$ for the same basis sets. In the last two rows, the values of the optimal values of $s$ for the $\mathrm{BBC}++$ functional and the corresponding values of $\alpha$ are also given.

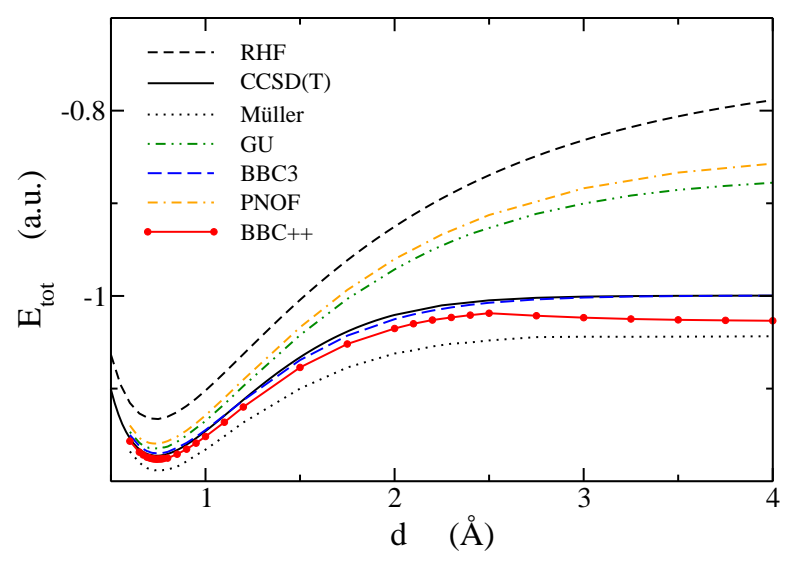

FIG. 2: Dissociation curve of the $\mathrm{H}_{2}$ molecule with different functionals of the 1-RDM.

to a large negative value for $\alpha$. While $\alpha$ has a value of -0.0388 at the equilibrium distance, it becomes -0.335 at a distance of $4 \AA$. As we can see from Fig. 1, this results in a negative value for $s$ driving the $\mathrm{BBC}++$ towards the Müller functional. Consequently, the functional overcorrelates and leads to a decrease in the total energy with increasing distance for the $\mathrm{H}_{2}$ molecule. It is worth mentioning that the dissociation of the $\mathrm{H}_{2}$ molecule is a rather difficult case for DFT functionals ${ }^{36,37}$.

Finally, a second pathology of the $\mathrm{BBC}++$ functional is its obvious size inconsistency: Consider a system consisting of two independent sub-systems, for example two finite systems at a large distance. If the two sub-systems are identical, the $\alpha$ of the total system is equal to the values of each sub-system. In the extreme case, however, where one of the sub-systems is much larger than the other, the common value for $\alpha$ is completely determined by the larger sub-system. That is, since the two systems are independent, the functional, when applied to the composite system, gives a different result for the smaller sub-system than when applied to the sub-systems independently.

\section{CONCLUSION}

We presented a RDMFT functional, which we call $\mathrm{BBC}++$, based on an idea to circumvent the dependence on the density parameter $r_{s}$ of functionals derived from the homogeneous electron gas. This idea is applied to the $s$-functional introduced in Ref. 28 . We apply BBC ++ in the calculation of correlation energies of small atomic and molecular systems and show that its performance is satisfactory. We also discuss pathologies of this functional, with the most important being its size inconsistency.

Despite its pathologies, the $\mathrm{BBC}++$ functional represents an important step in the development of 1-RDM functionals. It is a successful attempt to apply approximations originally developed for the HEG to finite systems. In other words, within RDMFT it is possible to develop functionals that perform equally well for extended systems, like the HEG, as well as small atomic and molecular systems. The present work serves as an initiative for the development of better approximations based on the HEG and, furthermore, their application to finite systems in the future.

\section{Acknowledgments}

This work was supported in part by the Deutsche Forschungsgemeinschaft within the program SPP 1145, by the EXCITING Research and Training Network, and by the EU's Sixth Framework Program through the Nanoquanta Network of Excellence (NMP4-CT-2004500198). 
1 M. A. Buijse, PhD Thesis, Vrije Universiteit Amsterdam (1991); M. A. Buijse, E. J. Baerends, Mol. Phys. 100, 401 (2002).

2 O. Gritsenko, K. Pernal, E. J. Baerends, J. Chem. Phys., 122, 204102 (2005).

3 S. Sharma, J. K. Dewhurst, N. N. Lathiotakis, E. K. U. Gross, arXiv:0801.3787.

4 T. L. Gilbert, Phys. Rev. B12, 2111 (1975).

5 A. M. K. Müller, Phys. Lett. A 105, 446 (1984).

6 S. Goedecker, C. J. Umrigar, Phys. Rev. Lett. 81, 866 (1998).

7 G. Csányi, S. Goedecker, T. A. Arias, Phys. Rev. A65 032510 (2002).

8 K. Yasuda, Phys. Rev. Lett. 88, 053001 (2002).

9 C. Kollmar, J. Chem. Phys. 121, 11581 (2004).

10 J. Cioslowski, K. Pernal, M. Buchowiecki, J. Chem. Phys. 119, 6443 (2003).

11 K. Pernal, J. Cioslowski, J. Chem. Phys. 120, 5987 (2004).

12 N. N. Lathiotakis, N. Helbig, and E. K. U. Gross, Phys. Rev. A 72, 030501 (2005).

13 J. Cioslowski and K. Pernal, Phys. Rev. B 71, 113103 (2005).

14 M. Piris, Int. J. Quant. Chem. 106, 1093 (2006).

15 C. Kollmar, J. Chem. Phys., 125, 084108 (2006).

16 M.A.L. Marques, N. N. Lathiotakis, Phys. Rev. A 77, 032509 (2008).

17 M. Piris, X. Lopez, and J.M. Ugalde, J:Chem. Phys. 126, 214103 (2007)

18 P. Leiva, and M. Piris, J. Chem. Phys., 123, 214102 (2005).

19 K. Pernal, J. Cioslowski, Chem. Phys. Lett., 412, 71 (2005).

20 P. Leiva and M. Piris, J. Mol. Struct.-Theochem., 770, 45
(2006)

21 P. Leiva and M. Piris, Int. J. Quant. Chem., 107, 1 (2007).

22 N. Helbig, N. N. Lathiotakis, M. Albrecht, E. K. U. Gross, Europhys. Lett. 77, 67003 (2007).

23 V. N. Staroverov and G. E. Scuseria, J. Chem. Phys. 117, 2489 (2002).

24 J. M. Herbert, J. E. Harriman, Chem. Phys. Lett. 382, 142 (2003).

25 N. N. Lathiotakis, M.A.L. Marques, J. Chem. Phys., 128, 184103 (2008)

26 J. Cioslowski and K. Pernal, J. Chem. Phys. 111, 3396 (1999).

27 G. Csányi, T. A. Arias, Phys. Rev. B61 (2000) 7348.

28 N. N. Lathiotakis, N. Helbig, E. K. U. Gross, Phys. Rev. B 75195120 (2007).

${ }^{29}$ D. M. Ceperley and B. J. Alder, Phys. Rev. Lett. 45, 566 (1980).

30 G. Ortiz, P. Ballone, Phys. Rev. B50, 1391 (1994); 56, 9970 (1997).

31 M. W. Schmidt, et al., J. Comput. Chem. 14, 1347 (1993).

32 T. H. Dunning, Jr. J. Chem. Phys. 90, 1007 (1989).

33 D. E. Woon and T.H. Dunning, Jr. J. Chem. Phys. 100, 2975 (1994).

34 J. A. Pople, M. Head-Gordon, and K. Raghavachari, J. Chem. Phys. 87, 5968 (1987).

35 Gaussian 03, Rev D.01, M. J. Frisch, et al, (Gaussian Inc., Wallingforth CT, 2004).

36 M. Grüning, O. V. Gritsenko, and E. J. Baerends, J. Chem. Phys. 118, 7183 (2003).

37 E. J. Baerends, Phys. Rev. Lett. 87, 133004 (2001). 